\title{
Polymeric Materials for Printed-Based Electroanalytical (Bio)Applications
}

\author{
Stefano Cinti \\ Department of Chemical Science and Technology, University of Rome "Tor Vergata", \\ Via della Ricerca Scientifica 1, 00133 Rome, Italy; stefano.cinti@uniroma2.it; Tel.: +39-06-7259-4411
}

Received: 23 October 2017; Accepted: 22 November 2017; Published: 24 November 2017

\begin{abstract}
Advances in design of selective interfaces and printed technology have mighty contributed to the expansion of the electroanalysis fame. The real advantage in electroanalytical field is the possibility to manufacture and customize plenty of different sensing platforms, thus avoiding expensive equipment, hiring skilled personnel, and expending economic effort. Growing developments in polymer science have led to further improvements in electroanalytical methods such as sensitivity, selectivity, reproducibility, and accuracy. This review provides an overview of the technical procedures that are used in order to establish polymer effectiveness in printed-based electroanalytical methods. Particular emphasis is placed on the development of electronalytical sensors and biosensors, which highlights the diverse role of the polymeric materials depending on their specific application. A wide overview is provided, taking into account the most significant findings that have been reported from 2010 to 2017.
\end{abstract}

Keywords: electroanalysis; printed electrodes; conductive polymers; biosensors

\section{Introduction}

Electroanalytical methods have gained a leading role in the implementation of user-friendly analytical devices [1]. The use of electroanalytical tools has been adopted worldwide because of the most known (and commercially successful) model of an easy-to-use device: the blood glucometer. It allows everyone, everywhere, to autonomously monitor the level of blood glucose (almost $90 \%$ of the entire biosensors global market is represented by this device) [2]. Only taking into account the Roche's plant in Indianapolis, over 2 billion test strips are produced per year [3].

Nowadays, self-testing devices represent the main request in the diagnostic field [4]. Inspired by the successful example reported above, the idea is to provide non-specialists with portable devices for many applications, from the environmental to the clinical, through the agri-food field. Electroanalysis, whether combined with printed technologies, i.e., screen-printing [5], inkjet printing [6], or wax printing [7], represents a promising starting point for reaching this goal. Its relevance is strictly bound to its operational simplicity and "blindness" towards colored/turbid solutions (real matrices often limit optical-based tests). The desired devices should satisfy the following requirements: they should be sensitive, small, lightweight, portable, disposable, and affordable. To deal with these, the printed electroanalytical plaftorms can be ad-hoc manufactured by using various supports for printing, i.e., plastic, ceramic, paper, removable tattoo, etc. [8-11]. Plenty of materials are adopted to make the devices sensitive and specific, i.e., metal nanoparticles, carbonaceous nano- and micro-materials, bioelements (enzyme, nucleic acids, antibodies, etc.), and polymers, etc. [12-15]. Depending on the specific chemical reactions that are involved/required to sense a target molecule, the use of some materials might fall within different scopes. For instance, gold nanoparticles (AuNPs) can serve as sensing elements (detection of metals in acidic solution, i.e., arsenic, mercury, copper, etc.) [16-18] or to create a recognition element/electrode interface, i.e., a DNA-based platform (AuNPs are used to link the electrode to the thiolated-DNA that represents the recognizing probe) $[19,20]$. 
Another class of "dual-use" materials is represented by polymers. However, the word "polymers" is too generic and the utilization of such materials is strictly dependent on what polymer is being utilized, and why. Among these, conductive polymers combine the conventional properties of polymers and the unique electronic properties that characterize metals and/or semiconductors [21,22]. The high $\pi$-conjugation of polyacetylene (PA), polypyrrole (PPy), polyaniline (PANI), and polythiophene (PTh), etc., makes them suitable for developing sensing platforms. Their doping may result in diverse materials: insulators, semi-conductors, or conductors [23,24]. They can be applied as conductivity enhancers (when combined with a "real" sensing element), or, alternatively, they can be employed as the sensing materials. For instance, PANI can be applied in a different way: combined with graphene and polyvinylpyrrolidone (PVP) [25], PANI led to an improvement of the conductivity in the biosensing of cholesterol, while Killard and his group utilized PANI to detect ammonia in the breath [26]. Beyond its conductive properties, electroanalysis also takes advantage of polymers for other purposes. Sometimes, polymers and/or mixtures of them can be utilized with the only aim of entrapping sensing elements, as in the case of glucose biosensor fabrication: Brett's group used a layer-by-layer approach combining the positively-charged chitosan together with the negatively-charged poly(styrene sulfonate) (PSS) [27], while Ayenimo and Adeloju entrapped the enzyme by electropolymerizing pyrrole on the electrode surface [28]. Another widely utilized polymer in electronalytical manufcaturing is Nafion: it is a perfluorinated sulphonated cation exchanger, highly permeable to cations. It is generally coated on the electrode surface to avoid interference from negatively-charged species and to enhance the accumulation of metals [29]. These are just some of the applications of polymers within electroanalysis.

In this review, readers will be provided with the main techniques that are used to modify printed electrodes with polymeric materials. The main modification approaches, i.e., drop casting, dispensing, inkjet printing, screen-printing, and electrodeposition, will be described, highlighting their strengths/weaknesses. Moreover, polymers will be grouped in three main sections: those that are involved as the sensing element, those that are utilized to improve the electrochemical performance of printed electrodes, and those that are employed to entrap/protect the (bio)sensing element.

\section{Techniques for Integrating Polymeric Materials onto Printed Devices}

Depending on (i) the experimental needs, (ii) the laboratory facilities, and (iii) the properties of the polymer, different techniques can be adopted to customize printed electrodes with polymeric materials. In particular, the modification of a certain substrate with polymeric materials depends on the polymer itself. Taking into account the polymer, many considerations should be taken into account: one of the principal issues related to monomers and polymers is their insolubility, especially in aqueous-based solutions. Even if their solubility might be improved by dissolving them in organic solvents, the use of plastic-based cartridges (as in the case of inkjet printing) could represent a limitation. Moreover, the post-processing approach is strictly dependent on the particular platform, as for the case of protecting polymers: a thermal polymerization is often not suitable for protecting an enzyme (it could be denatured). The approaches that have been reported in the literature during the 2010s are mainly focused on drop casting, inkjet printing, screen-printing, and electropolymerization. In this section, all these approaches are described, highlighting their characterizing features. To provide the reader with a quick overview, the main significant advantages and disadvantages of each technique are schematized in Figure 1. 


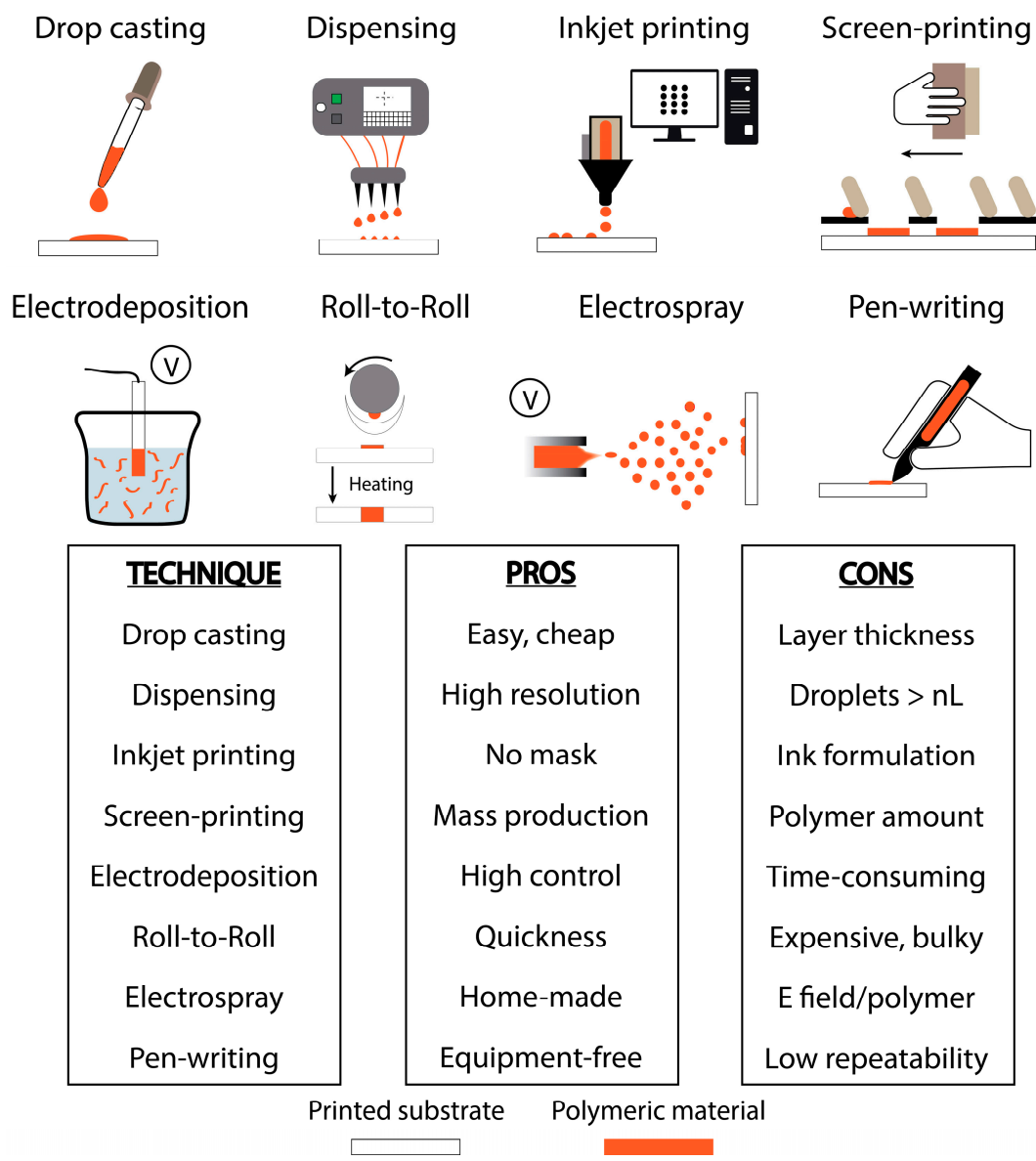

Figure 1. Scheme of the main utilized techniques that are used to modify printed electrochemical devices with polymeric materials.

Drop casting approach is without doubt the easiest and cheapest method to modify a surface. It allows one to modify the chosen surface in a single step, or with multi-steps, in order to choose the ideal amount of drop that fits with the area of the surface, i.e., electrode. Although this procedure does not require particular instrumentation (a pipette is enough), some considerations are needed: (i) water-insolubility of some polymers often limits their applicability, especially if screen-printed electrodes (SPEs) are modified (the conductive inks that are used in SPE fabrication could be removed by the addition of polymers that have been prepared in organic solvents); (ii) $\mu \mathrm{L}$-drops are subjected to "coffee-ring effect" that produces accumulation of the polymers at the borders of the drop [30], providing poor, homogenous, modified surfaces (reproducibility might be affected); and (iii) radial diffusion of the drop limits the modification of rounded surfaces. The same (enhanced) method can be adopted by using an automated dispenser, which can be thought of as a high-tech drop casting. Dispensers are used for multiple applications in the field of diagnostics: biosensors (glucose strips), dry chemistry (pH strips), lateral flow (pregnancy test), and low volume PCR (genomic research), etc. A world leader company is BioDot (www.biodot.com). Its non-contact technology can typically dispense fluids down to a volume of a $\mathrm{nL}$. However, to realize micro-array, $\mathrm{nL}$ droplets could not be enough. Another technology, widely used for manufacturing polymer microarray fabrication, is inkjet printing. This approach is classified as drop-on-demand. It requires an inkjet printer (Fujifilm Dimatix DMP-2831/2850 are the most commonly used ones on the small laboratory scale, while printers from Ceradrop, Pixdro, and Microfab are usually adopted on the large scale) that has a mechanism similar to those used in office environments; piezoelectric- and thermal-inkjet are the main utilized technologies [31,32]. The former generates a drop by deforming the jet chamber depending on the 
piezoelectric properties of the chamber wall, while the latter produces drops by heating and vaporizing the liquid in the jet chamber. Typically, vapor point of solvent can limit thermal-based technologies: in fact, those polymers that can be only dissolved in fast evaporating solvents might dry out the nozzles quickly. Even if inkjet printing allows one to deposit small droplets in the pL-nL range, the "coffee-ring effect" needs to be carefully evaluated: it can be adjusted by controlling experimental parameters such as drop spacing and temperature [33]. A big advantage is that inkjet printers do not require a mask: they offer the possibility of modifying all the geometric configurations, just by designing the ad-hoc pattern with a dedicated drawing software. However, viscosity and particle size can limit this approach. In particular, dimension of the polymers should be 100 fold smaller than the nozzle aperture diameter, otherwise nozzles can get easily clogged, i.e., a $10 \mu \mathrm{m}$ particle cannot be inkjet printed with a $20 \mu \mathrm{m}$ nozzle. This represents the main disadvantage of inkjet printing. To avoid the issues related to both size and solubility of polymers in aqueous solutions, and also those related to the realization of non-homogenous modified surfaces, polymers can be directly dispersed in the conductive ink and screen-printed onto strips [34,35]. Since the use of screen-printing is not widely reported for fabrication of polymeric-based electrochemical platforms, it owns its experimental advantages to the possibility of both manufacturing electrodes and manually integrating polymer in the conductive inks. However, its major limitation is due to its low resolution in creating micro-sized electrodes. Other limitations concern the integration of polymers within the conductive ink: (i) high amount of polymeric materials is required (typically in the order of grams); and (ii) if only the working electrode needs to be modified, a particular screen has to be utilized (it means longer steps). Switching from the mechanical (casting, printing) to the electrochemical procedures, electrodeposition represents a very common approach used in the modification of printed electrochemical strips with of polymers [36,37]. Usually, electropolymerization is carried out by applying a voltage that can be fixed (amperometry) or variable (voltammetry). This approach allows one to control the amount of deposited polymers, e.g., by selecting the duration of the applied voltage, adjusting the window of potential, varying electrochemical parameters such as number of scans, scan rate, etc., and even obtaining a more homogenous, coated working electrode with respect to the other methods. However, its limitations are related to its automation: common potentiostats allow the user to modify electrodes one-by-one, ensuring a longer time for multi-electrodes. Even if a parallel connection would lower the required time for multi-deposition of polymers, in practice the commercially available multi-output potentiostats are majorly connected in series. Even if these are the majorly utilized techniques for customizing printed electroanalytical platforms with polymer-based modifiers, other approaches can serve to accomplish this goal (although some techniques have not been applied to (bio)electrochemical detection yet); among these, roll-to-roll [38], electrospray [25], pen-writing [39], and dip coating [40] represent other possible methods. However, roll-to-roll allows a quick modification of a high amount of electrodes, but the equipment required is bulky and expensive; electrospray can be carried out with home-made apparatus, but one of its limitations could be ascribable to the interaction between the electric field applied to generate droplets and the polymer; pen-writing is also a very versatile way to produce or modify printed electrodes, but it lacks of repeatability; dip coating for layer-by-layer structuring of electrodes is a simple and effective approach, which exploits the adsorption of oppositely charged polymer layers, but it requires many steps.

\section{Role of Polymeric Materials in Printed-Electrochemical Devices}

\subsection{Polymers as the Sensing Element}

The first application related to the use of polymeric materials in the realization of electrochemical devices is devoted to those that have been used as a real sensing element. In particular, these materials are often realized by employing conductive polymers. These are particularly useful in the detection of small-molecules [41], due to their sensitivity to minor perturbations. In fact, gas-sensors developed by employing conductive polymers are widely known [42]. 
For instance, the group headed by Killard developed a human breath ammonia sensor by inkjet printing PANI onto a screen-printed silver interdigitated electrode [26], Figure 2A. This conducting polymer has been interrogated as ammonia-sensitive material due to a well-understood mechanism, $\mathrm{PH}^{+} \mathrm{A}^{-}+\mathrm{NH}_{3} \leftrightarrow \mathrm{P}+\mathrm{NH}_{4}{ }^{+} \mathrm{A}^{-}$, where $\mathrm{P} \mathrm{PH}^{+}$, and $\mathrm{A}^{-}$are the deprotonated polymer, the protonated polymer, and the dopant counter anion, respectively. The presence of ammonia leads to a deprotonation of the polymer, which produces a decrease in conductivity. They choose the electrochemical impedance spectroscopy (EIS) to correlate the level of ammonia with the conductivity decrease. PANI was chemically synthesized using dodecylbenzenesulfonic acid (DBSA) as the dopant and to avoid aggregation of PANI during the process [43]. During the optimization procedures for manufacturing the device, a $10 \mathrm{pL}$ printer cartridge with a drop spacing of $20 \mu \mathrm{m}$ was utilized. Authors quantified the impedimetric response to ammonia after 16 simulated breaths. A limit of detection equal to $4.1 \mu \mathrm{g} / \mathrm{m}^{3}$ has been calculated and a linearity in the range of $27-1514 \mu \mathrm{g} / \mathrm{m}^{3}$ has been observed. The effectiveness of PANI as sensing material have been also evaluated in presence of the major interfering species that can be found in air or breath: ammonia resulted as the only major gas to give a significant effect (compared to carbon dioxide, nitrogen, oxygen, hydrogen sulfide, and nitric oxide).

The sensing properties of PANI were also exploited for the detection of analytes in aqueous solution. Kit-Anan et al. [44] modified a disposable paper-based carbon-SPE with PANI to detect ascorbic acid. As for the ammonia sensor developed by Killard [26], PANI was inkjet-printed using a Dimatix Inkjet Printer (Fujifilm, Tokyo, Japan). In this case, PANI was chemically sinthesized using linear alkyl benzene sulphonic acid (LABSA) instead of DBSA. Authors evaluated the effect of printing different amount of PANI (1 to 10 layers): 5 layers provided the highest sensitivity towards ascorbic acid in comparison with the bare SPE. The high electrochemical redox catalytic properties of PANI towards ascorbic acid led to a $>10$-fold increase of the sensitivity with respect to bare SPE. Ascorbic acid was chronoamperometrically detected reaching a detection limit of $30 \mu \mathrm{M}$ and a linear range extended up to $270 \mu \mathrm{M}$. Their sensors compared positively, in terms of analytical performances, with the other existing electrochemical devices that exploit PANI as the sensing element. In addition, they evaluated the selectivity of the platform by performing measurements in presence of uric acid and acetoaminophen (two of the majorly occurring interferring species present in biological fluids): 500 and $140 \mu \mathrm{M}$ of uric acid and acetaminophen, respectively, did not produce detectable signals.

Bergamini and co-workers electropolymerized a synthetic polyaminoacid, poly-L-histidine ( $\mathrm{PH})$, onto a carbon-based SPE to detect isoniazid in human urine [36]. Authors compared the sensing effectiveness of the electropolymerized $\mathrm{PH}(\mathrm{EPH})$ with the drop cast $\mathrm{PH}$. EPH displayed a relevant electrocatalytic effect in terms of less negative peak-potential $(-0.89 \mathrm{~V}$ vs. $-1.02 \mathrm{~V})$ and higher peak intensity. Only in presence of the EPH film the electrocatalytic reduction of isoniazid was observed: the reduction potential observed at the bare SPE was the same as that in presence of drop cast PH. One possible explanation for this experimental behavior is the diverse isoniazid diffusion within the inner domain that reaches the working electrode surface: EPH allowed a better diffusion through the more opened structure of the film if compared to the thicker PH film formed by drop casting. Since the methodology for preparing the best sensing materials was set-up, the EPH-SPE was challenged in presence of isoniazid by using linear sweep voltammetry (LSV), differential pulse voltammetry (DPV), and square-wave voltammetry (SWV), reaching $5 \times 10^{-7}, 1.7 \times 10^{-7}$ and $2.5 \times 10^{-7} \mathrm{M}$ as the detection limit, respectively. Although this platform was challenged in a liquid matrix, the interferences were not evaluated.

PPy was used to screen a cancer biomarker in point-of-care configuration [37]. Carcinogenic embryonic antigen (CEA) protein, which is routinely used to follow-up the progression of specific cancer diseases, was chosen as the biomarker. In this case, the electropolymerized PPy was named "plastic antibody". Authors realized an imprinted polymer on the printed Ag-working electrode, as shown in Figure 2B. The biomimetic PPy film was formed by cycling the electrode immersed in an aqueous solution, which contained Py monomer and CEA. After the 15th cycle, the biomimetic film was formed. The biomarker molecules were initially entrapped in the polymeric network and 
successively removed by protease action. The recognition capability of the PPy "plastic antibody" was evalauted with diverse analytical techniques such as CV, EIS, and SWV. All the techniques gave a linearity response up to $1.25 \mathrm{pg} / \mathrm{mL}$ (against logarithm concentration). The non-imprinted PPy gave a non-specific response lower than those due to the "plastic antibody". The effectiveness of the platform was then evaluated in CEA-spiked urine, observing the same linearity range even if the slope was affected.

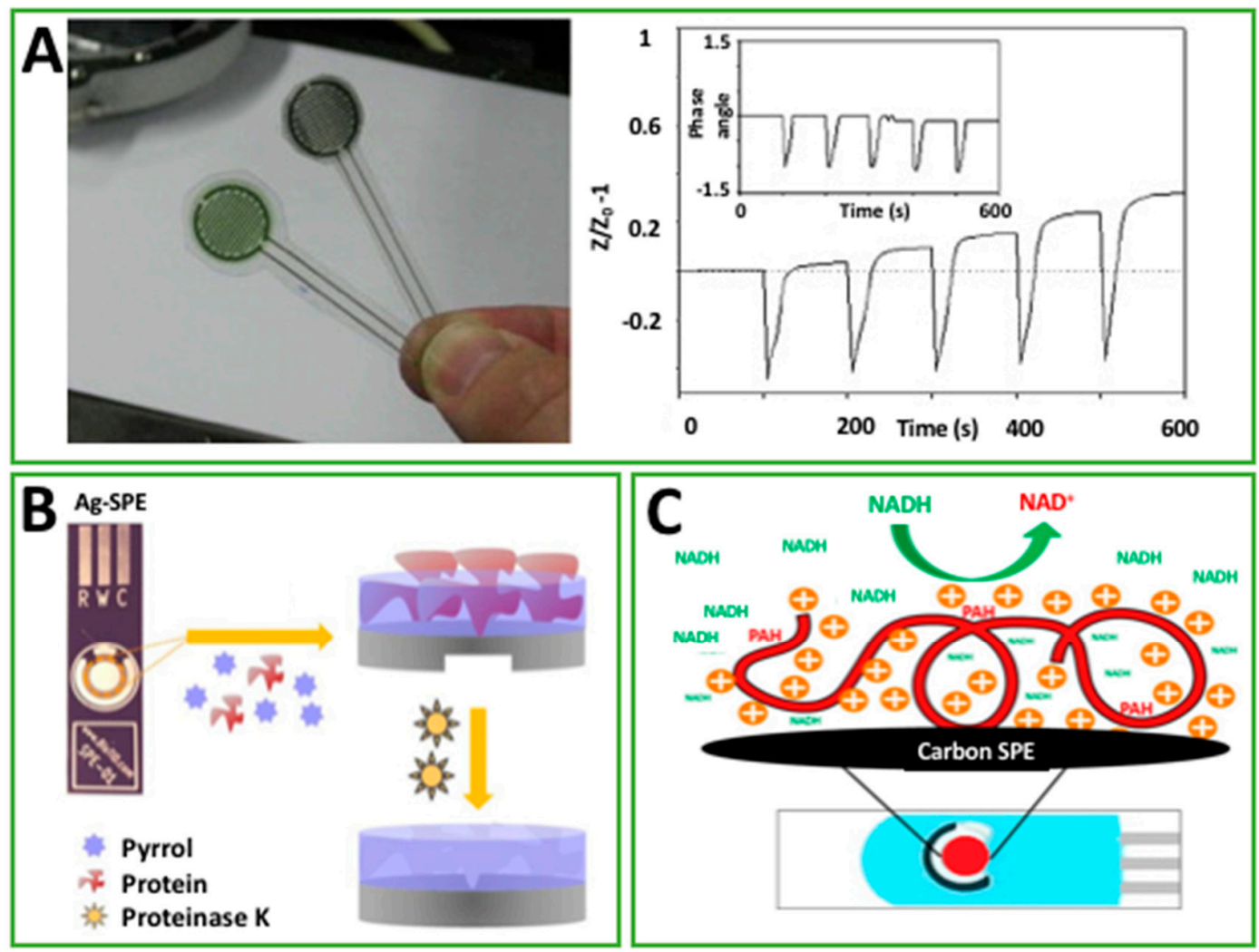

Figure 2. (A) Interdigitated polyaniline nanoparticle modified electrode. Screen-printed silver interdigitated electrodes were covered with multiple layers of an inkjet-printed polyaniline nanoparticle dispersion forming a green film for ammonia detection (Permission of [26]); (B) schematic representation of the two stages used in the synthesis of Ag- screen-printed electrode (SPE)_/-/ polypyrrole (PPy) devices, including the electropolymerization of Py in the presence of carcinogenic embryonic antigen (CEA), and the protein removal by proteinase K (Permission of [37]); (C) a SPE modified with poly(allylamine hydrochloride) for electrochemical detection of NADH (reduced nicotinamide adenine dinucleotide) (permission of [45]).

In 2014, Rotariu et al. [45] reported the utilization of a positively charged polyelectrolyte, poly(allylamine hydrochloride) (PAH), for the detection of NADH in carbon-based SPE, Figure 2C. The reason for choosing this polyelectrolyte was to exploit the electrostatic interaction with NADH, which can facilitate the accumulation at the SPE, producing a sensitivity enhancement. Positively charged PAH was drop cast onto the working electrode surface after a negatively charged surface was obtained by ending the cycling at a negative potential. The use of PAH-SPE led to an increase of the oxidation current from 4 (bare SPE) to $56 \mu \mathrm{A}$ (14-fold in presence of $5 \mathrm{mM} \mathrm{NADH}$ with cyclic voltammetry experiments). This results were obtained by optimizing the amount of PAH onto the SPE; in fact, they observed that a low concentration of PAH was consistent with a very low response, perhaps due to an unstable film formation. However, a overly high concentration of PAH increased the film thickness, which limited the diffusion of NADH inside the film, and consequently the sensitivity of the platforms. $0.5 \mathrm{mg} / \mathrm{mL}$ PAH was chosen as the optimal amount, and EIS experiments 
confirmed the effectiveness of PAH-SPE towards negatively charged molecules. The limit of detection of PAH-SPE was calculated as equal to $0.22 \mathrm{mM}$, with a linear range extended up to $5 \mathrm{mM}$. Even in presence of ascorbic acid, a negatively charged molecule, PAH, was capable to confer the system with a satisfactory selectivity.

Istamboulie et al. [34] directly screen-printed a PEDOT:PSS dispersion mixed with graphite paste in different ratios (from $20 \%$ to $100 \%$ polymer) and utilized this approach to develop an inhibition biosensor based on acetylcholinesterase (AChE) for the detection chlorpyrifos-oxon, an organophosphate insecticide. The PEDOT:PSS polymer was suitable for thiocholine oxidation (by-product of enzymatic reaction in presence of acetylthiocholine as the substrate), allowing the measurement of enzyme activity. Due to its intrinsic properties, 100\% PEDOT:PSS-ink was capable of improving $>2$-fold the conductivity with respect to the bare electrodes. Moreover, authors compared the analytical performance of their sensing polymer with those obtained by using cobalt phathalocyanine (CoPc), i.e., those classically used for thiocholine detection [46]. PEDOT:PSS was allowed to reach a detection limit as low as $4.4 \mathrm{nM}$ (considered as the chlorpyrifos-oxon concentration that induces a loss of signal of $5 \%$ ) after an incubation time of $10 \mathrm{~min}$.

Besides the variety of electrochemical sensors and biosensors that have been developed by employing a polymeric material as a sensing material, other approaches have been adopted. In particular, Dickert and Lieberzelt developed a plethora of platforms (principally based on quartz microbalance) with molecularly imprinted polymers (MIPs) to detect cells [47], bacteria [48], and viruses [49]. In addition, Lieberzelt and his group recently reported some electrochemical applications with MIPs: in 2014 [50], $\mathrm{Cu}^{2+}$ ions were conductometrically detected down to $0.2 \mathrm{mM}$ by polymerizing $N$-vinyl-2-pyrrolidone and, in 2016 [51], dengue virus was impedimetrically detected with a $0.12 \mathrm{pfu} / \mathrm{mL}$ detection limit by using a graphene oxide-polymer composite. Even the group of Cleij in Maastrischt, which is one of the most active regarding the use of MIPs, developed an impedimetric assay to detect histamine in intestinal fluid patient samples [52]. However, Cleij is a recognized expert on read-technology based on heat; accordingly, read-out technology conducting polymers are replaced by MIPs [53]. Moreover, a collaboration with Banks led to the innovative combination of thermal wave transport analysis (TWTA) and SPEs: MIPs were mixed within the conductive ink and successively screen-printed onto the substrate. TWTA was allowed to detect dopamine down to $0.26 \mu \mathrm{M}$, while cyclic voltammetry was allowed to reach $4.7 \mu \mathrm{M}$ as the detection limit [35].

\subsection{Polymers as Analytical Performances' Boosters}

In 2012, Quintana et al. [54] demonstrated the effectiveness of Nafion towards the development of bismuth-modified SPE to detect lead ions in milk. As reported previously by Wang, bismuth-film represented a "greener" approach to detecting heavy metals, avoiding the use of the more toxic electrodes based on mercury drops (hanging drop mercury electrodes, HDME) or films [55]. Bismuth is capable of forming alloys with metals, i.e., lead, cadmium, zinc, when these are dissolved in solution as cations, i.e., lead $(\mathrm{Pb})$ as $\mathrm{Pb}^{2+}$. Authors showed how the drop casting of Nafion onto the carbon-based SPE was consistent with a great improvement in sensitivity, up to a certain point. The modification of SPE with Nafion led to a sensitivity ehnancement towards lead ions, from $0.75 \mu \mathrm{AL} / \mu \mathrm{g}$ (un-modified bismuth-SPE) to 1.07 and to $1.1 \mu \mathrm{AL} / \mu \mathrm{g}$, respectively, using $0.5 \%$ and $1 \%(v / v)$ of Nafion, while the use of a more concentrated Nafion solution $(2 \%(v / v))$ produced a lower improvement of the sensitivity $(0.84 \mu \mathrm{AL} / \mu \mathrm{g})$. The presence of Nafion was capable of improving the sensitivity towards lead ions, but $2 \%(v / v)$ was consistent with a thick layer formed that hid the improvement. The developed procedures, adopting $0.5 \%(v / v)$ Nafion, allowed the authors to detect lead ions in contaminated milk at a concentration of $20 \mathrm{ppb}$ (legal limit). In addition to these findings, they utilized the same methods to evaluate the phytoremediation of an aquatic plant (Lemna minor) toward lead and cadmium ions, achieving satisfactory results: detection limit equal to 0.3 and $2 \mathrm{ppb}$ for lead and cadmium ions, respectively [56]. 
The use of a nanocomposite formed by graphene/polyvinylpyrrolidone/polyaniline (G/PVP/PANI) was effective in the realization of a paper-based device for amperometric cholesterol detection in human serum [25]. The paper-based platform was modified by electrospraying the final nanocomposite (Figure 3A). The electrocatalytic effect of the modifiers was estimated step-by-step, taking into account the role of each modifier. To do this, authors studied the platform with experiments of cyclic voltammetry in presence of ferri/ferrocyanide as the redox probe. While the presence of PANI only led to an increase of the current, the addition of graphene (as G/PANI) led to a net increase of the peak intensities and decrease of $\Delta \mathrm{E}$, indicating an electrocatalytic effect ( $\Delta \mathrm{E}$ significantly decreases from 0.508 to $0.155 \mathrm{~V}$, respectively, for PANI and G/PANI-modified SPE). Even in the previous case, the presence of $\mathrm{G}$ in the nanocomposite boosted the electron transfer kinetics of the platform. However, the authors highlighted how the presence of a small amount of PVP, forming the ultimate G/PVP/PANI composite, improved the dispersibility of G, increasing the electrochemical conductivity of electrodes, i.e., it was consistent with an ehnancement of the current peaks. Cholesterol was indirectly quantified with this electrochemical platform by monitoring the hydrogen peroxide by-produced from cholesterol oxidation in presence of cholesterol oxidase; G/PVP/PANI produced a dramatic increase of sensitivity (40-fold) in comparison with the bare SPE. Finally, cholesterol was detected down to a limit of $1 \mu \mathrm{M}$ in a linear range between 0.05 and $10 \mathrm{mM}$.

Another approach to detect ammonia was developed by Seekaew and colleagues [57]. Also, in this case, an interdigitated silver electrode was screen-printed, but the sensing layer was composed of a poly(3,4-ethylenedioxythiophene):poly(styrenesulfonate) (PEDOT:PSS) film. Authors inkjet-printed this conducting polymer by using a modified office printer (HP deskjet 2000 j210, Hewlett-Packard, Palo Alto, CA, USA) and filling the original cartridge with the desired modifier, Figure 3B. In addition, they also evaluated the combination of PEDOT:PSS with graphene: 8 printed layers were adopted to produce ca. $400 \mathrm{~nm}$ thick films. As already discussed for the sensing mechanism with PANI, the change in conductivity of PEDOT:PSS, as a consequence of the neutral polymer formation in presence of ammonia, has been exploited to correlate change of sensor resistance and ammonia concentration. The use of graphene in combination with PEDOT:PSS allowed the authors to improve the conductivity by more than a factor of two (compared with the pristine PEDOT:PSS), and it produced a sensor with a high selectivity to ammonia in the $25-1000$ ppm range, with a detection limit $<10$ ppm, even if the dynamic range toward ammonia was similar using the pristine film. Moreover, authors claimed that the addition of graphene had the effect of improving the specific adsorption on working area of the flexible sensor; response to ammonia appeared higher than those in presence of diethylamine, acetone, ethanol, toluene, and methanol. The same research group took advantage of the same sensing film to develop an amperometric glucose biosensor [58]. In this case, a carbon-based SPE was modified by drop casting a few microliters of the hybrid nanocomposite (glucose oxidase and graphene-PEDOT:PSS). In presence of glucose, the nanocomposite was capable of directly quantifying the electron transfer between glucose oxidase and the SPE, which was not achievable with the bare SPE. Although the linearity of the developed platform was obtained only from 20 to $900 \mu \mathrm{M}$ (not sufficient for diagnostic applications for the management of glucose-related diseases, i.e., diabetes), a detection limit of $0.3 \mu \mathrm{M}$ was appreciated.

Another example showing a polymer for improving electrochemistry is centered on PPy. It is one of the most used conducting polymers because of its good biocompatibility and mechanical properties, and it has been adopted to modify commercial, disposable electrochemical printed chip, to produce an impedimetric immunosensor for human chorionic gonadotropin (hCG) detection [59]. PPy was electro-grafted in presence of a copolymer, represented by the poly pyrrole-2-carboxylic acid (PPa), Figure 3C. Authors combined the features of these two polymers in order to develop a highly stable and sensitive immunosensor; while PPy is exploited for its high conductivity and biocompatibility, PPa is capabe of enhancing the hydrophilicity of the system and its binding ability towards hCG antibody. The ratio of PPy and PPa was chosen equal to 3:1 due to the best electroactivity provided. The effectiveness of this co-polymer electroactive platform allowed us to obtain a label-free impendance 
immunosensor with a detection limit of $2.3 \mathrm{pg} / \mathrm{mL}$ (with a sensor area of $2.64 \mathrm{~mm}^{2}$ ) and linearity range from 0.1 to $1 \mathrm{ng} / \mathrm{mL}$.

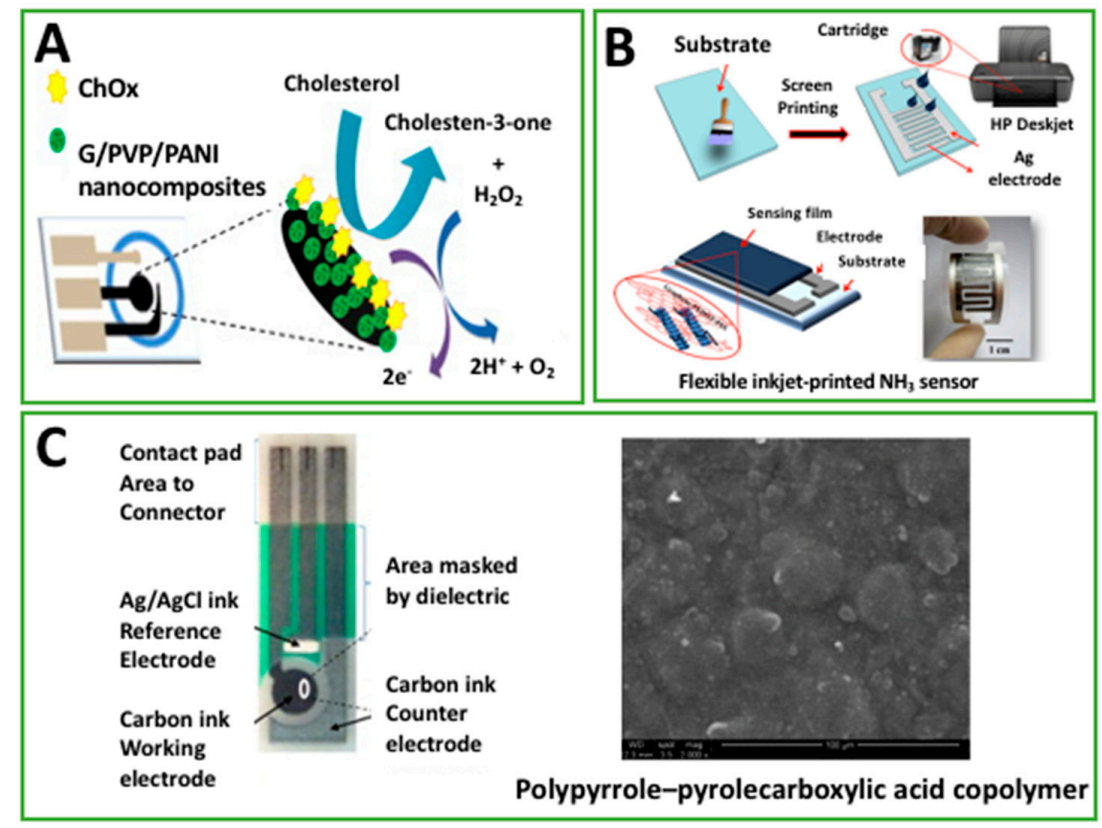

Figure 3. (A) The enzymatic reaction between cholesterol and ChOx on Graphene/polyvinylpyrrolidone (PVP)/polyaniline (PANI) modified, paper-based biosensor (permission of [25]; (B) schematic diagram of fabrication of a flexible-printed graphene-poly(3,4-ethylenedioxythiophene):poly(styrenesulfonate) (PEDOT:PSS) gas sensor for ammonia detection (permission of [57]); (C) Figure 3: the structure of disposable electrochemical printed (DEP) chip used within this work and SEM micrograph of deposited PPy-PPa copolymer film onto carbon ink working electrode (permission of [59]).

\subsection{Polymers to Entrap/Protect the (Bio)Sensing Element}

Xin et al. [60] developed a novel and disposable biosensor for determination of hydrogen peroxide by modifying a carbon-based SPE with a mixture made of $\mathrm{Fe}_{3} \mathrm{O}_{4}$-Au magnetic nanoparticles coated with horseradish peroxidase (HRP) and a graphene-Nafion film. Authors decided to use Nafion to stabilize and disperse graphene into an aqueous solution. The issue was due to difficulty of graphene in forming a uniform dispersion into an aqueous-based media. Due to its capability for film formation, nontoxicity, biocompatibility, mechanical robustness, and satisfactory water permeability, Nafion was chosen to form a homogenous dispersion of graphene, and also to better immobilize the enzyme (HRP). The amount of Nafion was optimized in order to achieve the best results in term of sensitivity and stability of the bio-platform: $10 \mu \mathrm{L}$ of graphene/Nafion was selected as the optimal amount of SPE modifier: a higher amount was consistent with a broadening of voltammogram and decrease of sensitivity. The presence of Nafion as the stabilizer within the modifiers' cocktail led to a current response that retained nearly $90 \%$ of its initial value over four weeks of storage. The platform was allowed to detect hydrogen peroxide with just three seconds amperometry, with a detection limit of $12 \mu \mathrm{M}$ and a linearity in the range between 0.02 and $2.5 \mathrm{mM}$.

Talarico and colleagues utilized chitosan (CS) as the dispersing agent of carbon black (CB) and acetylcholinesterase enzyme (AChE) to fabricate an organophosphorus pesticide biosensor with a "one step" procedure [61], as reported in Figure 4A. The choice of CS is linked to two main aspects: (1) it avoided the aggregation of $C B$ without the necessity of using ultrasounds to make a CB dispersion; and (2) it provided a biocompatible and favorable microenvironment for AChE immobilization, improving operational stability. This work represented the first example in which this bio-polymer has been employed for a single step procedure: in fact, CS has been usually adopted for 
enzyme immobilization using multi-step procedures [52,62]. CS was capable of providing a stable dispersion composed by AChE, which might be irreversibly inhibited by organophosphorus pesticides and $\mathrm{CB}$, which provided an electrocatalytic modifier to follow-up the detection of the enzymatic by-product. Authors experienced a worsening of the electrochemical behavior when the amount of CS was increased, probably due to its insulator nature: $0.05 \% w / w$ CS was selected as the best compromise in terms of sensitivity and stability of the platform. The printed device was capable, with $20 \mathrm{~min}$ as the inhibition time, of detecting paraoxon (taken as model compound) down to $0.05 \mathrm{ppb}$ in a linear range of $0.1-0.5 \mathrm{ppb}$. Moreover, the stability of the biosensor was evaluated in terms of adhesion of the CS-based mixture; five measurements after a period of $20 \mathrm{~min}$ in stirring conditions were consistent with a relative standard deviation $<8 \%$, and it was ascribable to the presence of CS as the mixture linker.

Loaiza et al. [53,63] utilized a combination of polyethyleneimine (PEI) and glutaraldehyde (GA) to covalently immobilize lactate oxidase (LOx) onto a SPE modified with platinum nanoparticles (PtNPs), supported on graphitized carbon nanofibers (PtNps/GCNF). The mechanism of the stabilization was ascribable to cross-linking between GA and the absorbed PEI layer. Authors reported that the presence of PEI and GA layers was necessary to retain the enzyme and get more sensitive and stable signals; in fact, while the PtNps/GCNF-PEI-GA-LOx-Gly-SPE displayed the highest amperometric signal in presence of lactate, the lowest sensitivity was obtained by PtNps/GCNF-LOx-Gly. Moreover, the presence of the polymeric network allowed the biosensor to retain $90 \%$ of the amperometric signal after 50 measurements. The biosensor gave a linear repsonse between 10 and $2000 \mu \mathrm{M}$, with a detection limit $<7 \mu \mathrm{M}$. Moreover, it was applied for measurements of lactic acid in alcoholic beverages, i.e., wine, cider. One of the most interesting aspects of their research was related to effect of PEI-GA over the storage stability; the sensitivity remains almost constant $(\geq 90 \%)$ after three months at room temperature, and after more than 18 months at $-20^{\circ} \mathrm{C}(\geq 95 \%)$.

In 2014, Karim et al. [40] took advantage of the negatively charged poly(sodium styrenesulfonate) (PSS) electrolyte to stabilize gold nanocubes (AuNC) in a biosensor architecture to detect cathecol. In particular, a layer-by-layer approach was followed. The process was performed by alternatively dipping a SPE in solutions containing positively charged AuNC-hexadecyltrimethylammonium (CTAB) and negatively charged PSS, Figure 4B. As the final step, a solution containing tyrosinase enzyme was dipped onto the modified SPE. The main role of PSS was to incorporate and stabilize the AuNC in the biosensing structure; instead, tyrosinase was electrostatically immobilized onto the positively charged AuNC-CTAB layer of the sensing architecture. Even though catechol was detected in a wide range of concentrations (10 nM-80 mM) with a detection limit of $0.4 \mathrm{nM}$, and the biosensor was applied for measurements in real tea samples, there was still the remaining challenge: long term stability. In fact, the stability was interrogated after several days of storage in buffer solution, and after 9 days the biosensor retained $83 \%$ of its initial activity. Probably, to enhance the storage stability that is almost entirely dependent on the enzyme activity, it could be enclosed into the multilayer structure to create a more effective environment, maybe employing a positively charged polymer (because of the negative charges in tyrosinase).

Another example of the utilization of a polymeric material to encapsulate a biorecognition element, i.e., an enzyme, was proposed by Weng et al. [64]; they produced a platform by inkjet printing formulations of PPy nanoparticles and enzymes onto SPE. In their study, the authors examined the behavior of two bio-hybrid composites: PPy-HRP and PPy-GOx. Moreover, to prevent the enzyme leaching out from the PPy film, they jetted a permselective ethyl cellulose membrane onto the testing area. The two hybrid nanocomposites were interrogated towards hydrogen peroxide and glucose detection, respectively, for PPy-HRP and PPy-GOx. The entrapment of enzymes within the polymeric matrix led to the detection of hydrogen peroxide from $10 \mu \mathrm{M}$ to $10 \mathrm{mM}$, and glucose from 1 to $5 \mathrm{mM}$. Long term stability studies indicated that PPy-HRP biosensor was capable of retaining more than $>90 \%$ of its initial sensitivity after a month $\left(\mathrm{dry}, 4^{\circ} \mathrm{C}\right)$. This stability was attributed to both the presence of PPy nanoparticles and the cellulose membrane, which minimized the loss of HRP enzyme, providing 
a kind of protection towards the enzyme. However, the possibility of extending this protection to GOx did not provide the same findings ( $<70 \%$ activity retention); the authors were not capable of providing an explanation, and they concluded that further studies were needed.

Shimomura and co-workers utilized another kind of polymer, a photo-cross-linkable one [65]. In particular, a polyvinil alcohol matrix, namely O-391, was used to entrap a mixture based on 3-nicotinamide adenine dinucleotide $\left(\mathrm{NAD}^{+}\right)$and $\beta$-hydroxybutyrate dehydrogenase (3HBDH), previously immobilized onto mesoporous silica, with the aim of developing a disposable amperometric biosensor for ketone 3- $\beta$-hydroxybutyrate (3HB), as reported in Figure $4 \mathrm{C}$. The SPE was modified with a Meldola's Blue layer which was responsible for the detection of the reduced NADH (as a consequence of the enzymatic oxidation of $3 \mathrm{HB}$ catalyzed by $3 \mathrm{HBDH})$. The response of the optimized biosensor had linear results, with substrate levels up to $8 \mathrm{mM}$ and a detection limit lower than $30 \mu \mathrm{M}$. The great advantage of this method was linked to the high storage stability that was exhibited. The biosensor was stored desiccated at $4{ }^{\circ} \mathrm{C}$ when not in use and, despite the dry conditions, it maintained more than the $90 \%$ of its initial enzyme activity over a period of six months.
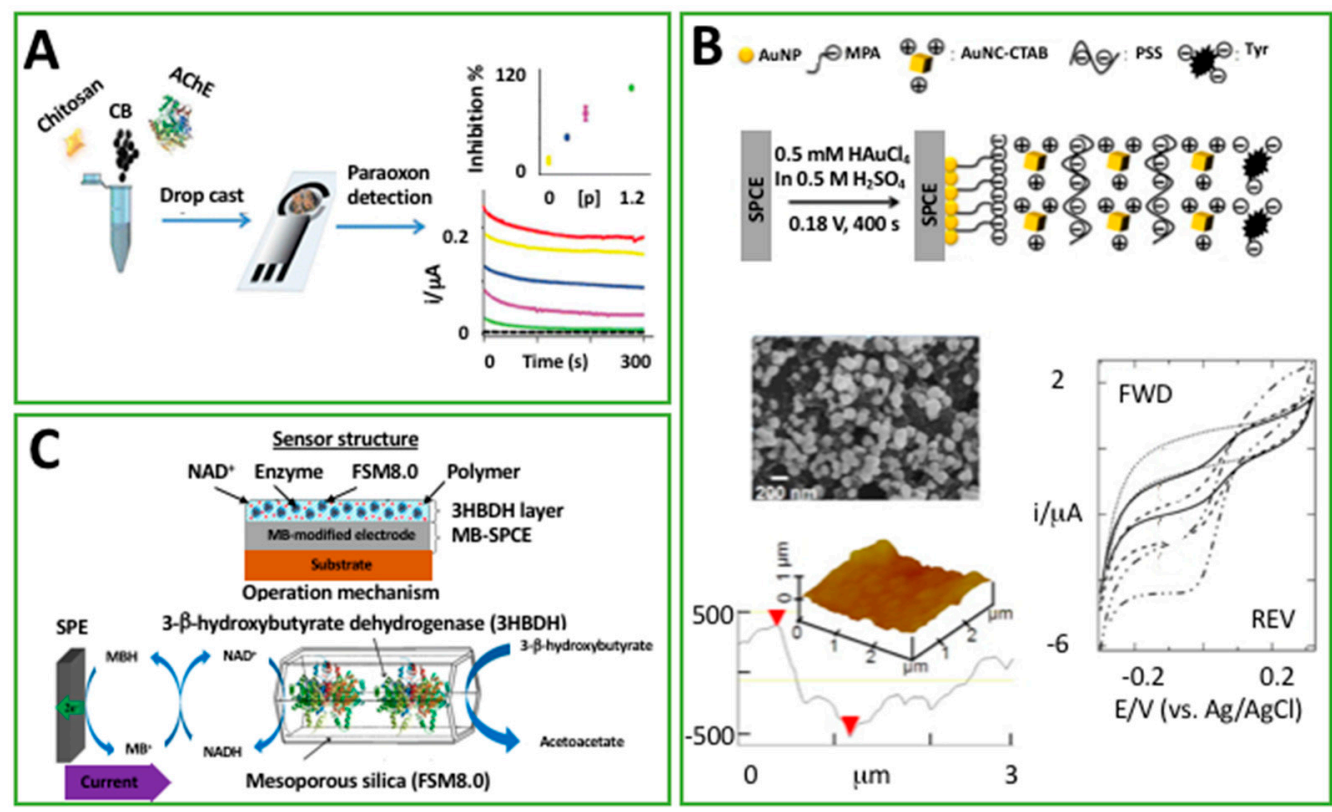

Figure 4. (A) Biosensors based on screen-printed electrodes modified with Acetylcholinesterase, carbon black, and chitosan for organophosphorus pesticide detection (permission of [61]); (B) schematic diagram showing the layer by layer process on an gold nanoparticles (AuNP)-deposited SPE, including SEM and AFM (Atomic Force Microscopy) images alongside the height profile of the platform. Representative cyclic voltammograms for different concentrations of catechol using the Tyrosinase-modified platform (permission of [40]); (C) layer structure and operation mechanism of 3HB biosensor (Permission of [65]).

Even the group headed by Girault exploited photo-polymerization to protect the sensing agent-carbon nanotubes (CNTs), in this case [66]. They combined the UV photo-polymerization to inkjet printing. Briefly, polyacrylamide was firstly inkjet-printed onto a device composed by Ag, $\mathrm{CNTs}$, and insulator (all of them were previously inkjet-printed). Successively, the polyacrylamide layer was irradiated by UV light, and a protecting layer was formed. Authors utilized their stand-alone electrochemical device for the detection of antioxidants in complex matrices such as untreated orange juice and red wine. Compared to the bare CNT electrode, as in the case of undiluted red wine, the presence of polyacrylamide hydrogel allowed us to compensate for the matrix effect issues which, as claimed by the authors, are often addressed by diluting and/or filtering real matrices. 
In order to provide the readers with a quick summary of the polymer-based devices that have been discussed in this compact review, Table 1 contains some of the most relevant information in terms of the sensing elements and the analytical performances of the investigated electroanalytical devices.

Table 1. Summary of the electrochemical-based (bio)sensors that exploit the presence of a polymeric material in their architecture.

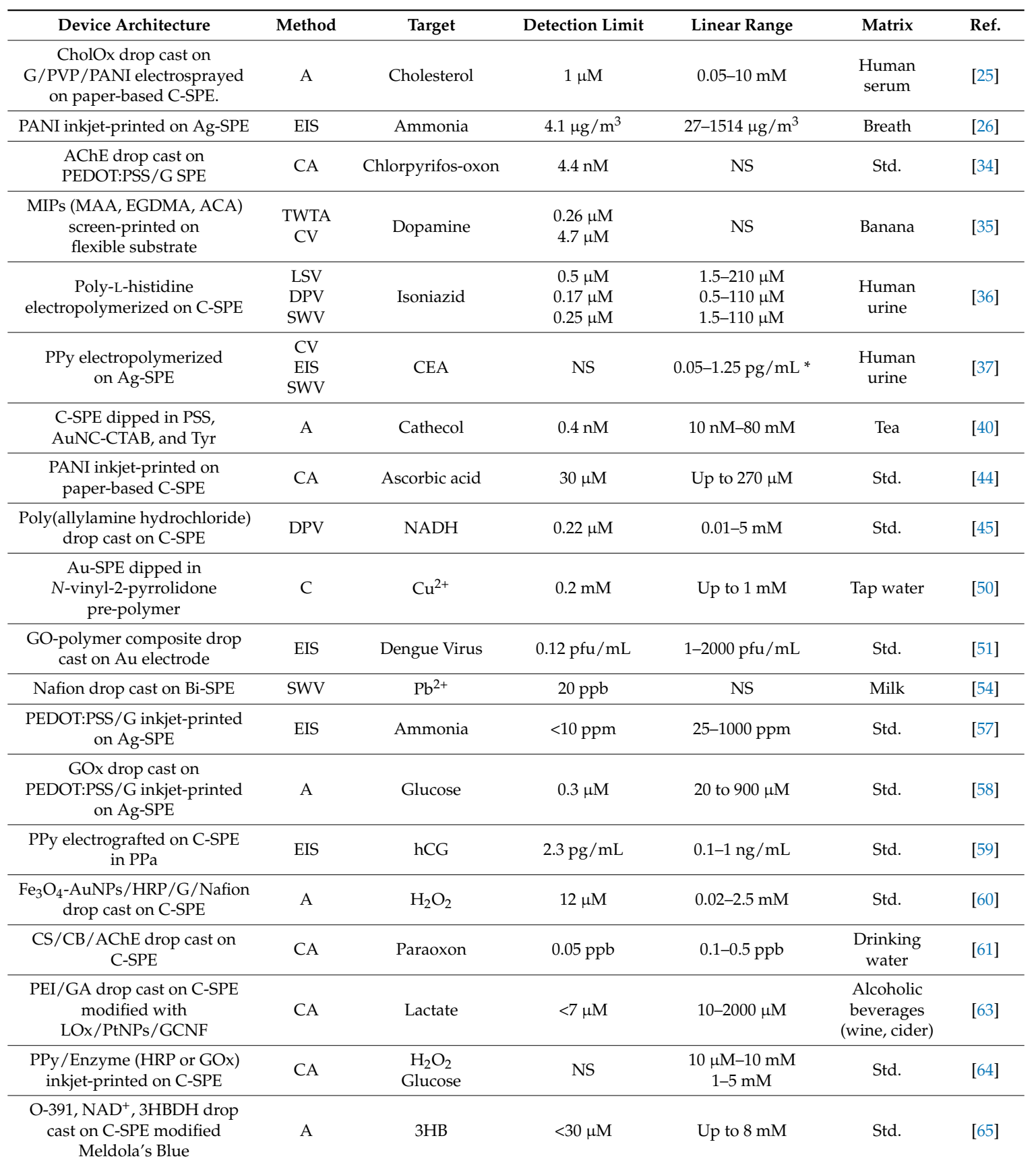

Symbols and abbreviations: NS: not specified; *: logarithmic scale; Std.: Standard solution; C-SPE: Carbon-SPE; Bi-SPE: Bismuth-SPE; Au-SPE: Gold-SPE; Ag-SPE: Silver-SPE; GE: Gold Electrode; PANI: Polyaniline; PPy: Polypyrrole; EIS: Electrochemical Impedance Spectroscopy; CA: Chronoamperometry; LSV: Linear Sweep Voltammetry; DPV: Differential Pulse Voltammetry; SWV: Square Wave Voltammetry; CV: Cyclic Voltammetry; C: Conductometry; TWTA: Thermal Wave Transport Analysis; A: Amperometry; G: Graphene; GO: Graphene oxide; GCNF: graphitized carbon nanofibers; PPa: Poly pyrrole-2-carboxylic acid; GA: Glutaraldehyde; MAA: Methacrylic acid; EGDMA: Ethylene glycol dimethacrylate; ACA: 4,4'-azobis(4-cyanovaleric acid); AChE: Acetyl cholinesterase enzyme; HRP: Horseradish peroxidase: GOx: Glucose oxidase; CholOX: Cholesterol oxidase; LOx: Lactate oxidase; Tyr: Tyrosinase; CEA: Carcinogenic embryonic antigen; hCG: Human chorionic gonadotropin; 3HBDH: $\beta$-hydroxybutyrate dehydrogenase; $3 \mathrm{HB}$ : Ketone 3 - $\beta$-hydroxybutyrate. 


\section{Conclusions}

After having reported some of the most successful applications of polymeric materials in combination with printed electrodes, it can be concluded that they offer a wide range of applications in the field of printable sensors and biosensors. This review has been divided in three main sections, depending on the major exploitation of the generally termed "polymers". This general term includes a plethora of different materials, i.e., conductive polymers, charged polymers, insulators, bio-polymers, and biomimetic "plastic antibodies", etc. Depending on the selected application and the specific need, a polymeric material can provide different features. In particular, the use of conductive polymers represents a relevant step forward towards the development of electrochemical platforms that gain the presence of a conductive backbone and a network that can stabilize the modifiers used in the chosen architecture. Some materials, i.e., chitosan, Nafion, demonstrated high efficacy in allowing nanomaterials i.e., metallic nanoparticles, carbonaceous, to form homogenuos dispersions that are often difficult to obtain. Moreover, the possibility of creating hybrid structures by entrapping enzymes is an added value toward the realization of stable platforms; in some cases, the reported shelf life reached 18 months. Storage stability is of crucial importance, especially for commercial applications. Even if the use of polymeric materials in combination with printed electrochemical strips has highlighted that polymer science might effectively enhance the experimental performance and stability of such methods, their utilization as sensing elements has not been very impressive. The examples related to the utilization of polymers as sensing elements are restricted to conductive polymers; however, their main drawbacks are usually due to a lack of high selectivity and the suitability of sensing prevalent, small molecules, i.e., ammonia, oxygen, and water, etc. In the case of molecular imprinted polymers, instead, the non-specific interactions between target and platforms can affect the reliability of the method. However, polymer-based modifiers can exhibit their effectiveness, especially in those electrochemical systems that necessitate a linker between the sensing (bio)element and the transducer. In addition, their inexpensiveness has to be positively taken into account during the development of printed platforms that generally are involved in mass-production processes. Future development could enable the combination of different technologies to realize the development of all-in-one diagnostic tools (not only within the electroanalytical field). Screen-printing for electrodes manufacturing and inkjet printing as the customizing tool might represent an ideal starting point for the realization of fully-printed low-cost devices.

Acknowledgments: S.C. acknowledges Fondazione Umberto Veronesi for the Post-Doctoral Fellowship 2017.

Conflicts of Interest: The author declares no conflict of interest.

\section{References}

1. Wang, J. Analytical Electrochemistry, 3rd ed.; John Wiley \& Sons: Hoboken, NJ, USA, 2006.

2. Turner, A.P. Biosensors: Sense and sensibility. Chem. Soc. Rev. 2013, 42, 3184-3196. [CrossRef] [PubMed]

3. Report: Roche Mulls Sale of Blood-Glucose Monitor Business. Available online: https://www.ibj. com/articles / 41392-report-roche-mulls-sale-of-blood-glucose-monitor-business? v=preview?v=preview (accessed on 18 October 2017).

4. Urdea, M.; Penny, L.A.; Olmsted, S.S.; Giovanni, M.Y.; Kaspar, P.; Shepherd, A.; Wilson, P.; Dahl, C.A.; Buchsbaum, S.; Moeller, G.; et al. Requirements for high impact diagnostics in the developing world. Nature 2006, 444, 73-79. [CrossRef] [PubMed]

5. Metters, J.P.; Kadara, R.O.; Banks, C.E. New directions in screen printed electroanalytical sensors: An overview of recent developments. Analyst 2011, 136, 1067-1076. [CrossRef] [PubMed]

6. Komuro, N.; Takaki, S.; Suzuki, K.; Citterio, D. Inkjet printed (bio)chemical sensing devices. Anal. Bioanal. Chem. 2013, 405, 5785-5805. [CrossRef] [PubMed]

7. Renault, C.; Koehne, J.; Ricco, A.J.; Crooks, R.M. Three-dimensional wax patterning of paper fluidic devices. Langmuir 2014, 30, 7030-7036. [CrossRef] [PubMed] 
8. Taleat, Z.; Khoshroo, A.; Mazloum-Ardakani, M. Screen-printed electrodes for biosensing: A review (2008-2013). Microchim. Acta 2014, 181, 865-891. [CrossRef]

9. Ahmed, M.U.; Hossain, M.M.; Safavieh, M.; Wong, Y.L.; Rahman, I.A.; Zourob, M.; Tamiya, E. Toward the development of smart and low cost point-of-care biosensors based on screen printed electrodes. Crit. Rev. Biotechnol. 2016, 36, 495-505. [CrossRef] [PubMed]

10. Arduini, F.; Cinti, S.; Scognamiglio, V.; Moscone, D. Paper-Based Electrochemical Devices in Biomedical Field: Recent Advances and Perspectives. Compr. Anal. Chem. 2017, 77, 385-413.

11. Bandodkar, A.J.; Jia, W.; Wang, J. Tattoo-Based Wearable Electrochemical Devices: A Review. Electroanalysis 2015, 27, 562-572. [CrossRef]

12. Siangproh, W.; Dungchai, W.; Rattanarat, P.; Chailapakul, O. Nanoparticle-based electrochemical detection in conventional and miniaturized systems and their bioanalytical applications: A review. Anal. Chim. Acta 2011, 690, 10-25. [CrossRef] [PubMed]

13. Wang, J. Carbon-nanotube based electrochemical biosensors: A review. Electroanalysis 2005, 17, 7-14. [CrossRef]

14. Cinti, S.; Arduini, F. Graphene-based screen-printed electrochemical (bio)sensors and their applications: Efforts and criticisms. Biosens. Bioelectron. 2017, 89, 107-122. [CrossRef] [PubMed]

15. Grieshaber, D.; MacKenzie, R.; Voeroes, J.; Reimhult, E. Electrochemical biosensors-sensor principles and architectures. Sensors 2008, 8, 1400-1458. [CrossRef] [PubMed]

16. Cinti, S.; Politi, S.; Moscone, D.; Palleschi, G.; Arduini, F. Stripping analysis of As(III) by means of screen-printed electrodes modified with gold nanoparticles and carbon black nanocomposite. Electroanalysis 2014, 26, 931-939. [CrossRef]

17. Cinti, S.; Santella, F.; Moscone, D.; Arduini, F. $\mathrm{Hg}^{2+}$ detection using a disposable and miniaturized screen-printed electrode modified with nanocomposite carbon black and gold nanoparticles. Environ. Sci. Pollut. Res. 2016, 23, 8192-8199. [CrossRef] [PubMed]

18. Wang, J.; Bian, C.; Tong, J.; Sun, J.; Xia, S. Simultaneous detection of copper, lead and zinc on tin film/gold nanoparticles/gold microelectrode by square wave stripping voltammetry. Electroanalysis 2012, 24, 1783-1790. [CrossRef]

19. Du, M.; Yang, T.; Jiao, K. Immobilization-free direct electrochemical detection for DNA specific sequences based on electrochemically converted gold nanoparticles/graphene composite film. J. Mat. Chem. 2010, 20, 9253-9260. [CrossRef]

20. Lu, J.; Ge, S.; Ge, L.; Yan, M.; Yu, J. Electrochemical DNA sensor based on three-dimensional folding paper device for specific and sensitive point-of-care testing. Electrochim. Acta 2012, 80, 334-341. [CrossRef]

21. Weng, B.; Shepherd, R.L.; Crowley, K.; Killard, A.J.; Wallace, G.G. Printing conducting polymers. Analyst 2010, 135, 2779-2789. [CrossRef] [PubMed]

22. Nambiar, S.; Yeow, J.T. Conductive polymer-based sensors for biomedical applications. Biosens. Bioelectron. 2011, 26, 1825-1832. [CrossRef] [PubMed]

23. Massonnet, N.; Carella, A.; de Geyer, A.; Faure-Vincent, J.; Simonato, J.P. Metallic behaviour of acid doped highly conductive polymers. Chem. Sci. 2015, 6, 412-417. [CrossRef] [PubMed]

24. Kaur, G.; Adhikari, R.; Cass, P.; Bown, M.; Gunatillake, P. Electrically conductive polymers and composites for biomedical applications. RSC Adv. 2015, 5, 37553-37567. [CrossRef]

25. Ruecha, N.; Rangkupan, R.; Rodthongkum, N.; Chailapakul, O. Novel paper-based cholesterol biosensor using graphene/polyvinylpyrrolidone/polyaniline nanocomposite. Biosens. Bioelectron. 2014, 52, 13-19. [CrossRef] [PubMed]

26. Hibbard, T.; Crowley, K.; Killard, A.J. Direct measurement of ammonia in simulated human breath using an inkjet-printed polyaniline nanoparticle sensor. Anal. Chim. Acta 2013, 779, 56-63. [CrossRef] [PubMed]

27. Barsan, M.M.; David, M.; Florescu, M.; Țugulea, L.; Brett, C.M. A new self-assembled layer-by-layer glucose biosensor based on chitosan biopolymer entrapped enzyme with nitrogen doped graphene. Bioelectrochemistry 2014, 99, 46-52. [CrossRef] [PubMed]

28. Ayenimo, J.G.; Adeloju, S.B. Amperometric detection of glucose in fruit juices with polypyrrole-based biosensor with an integrated permselective layer for exclusion of interferences. Food Chem. 2017, 229, 127-135. [CrossRef] [PubMed]

29. Amini, N.; Gholivand, M.B.; Shamsipur, M. Electrocatalytic determination of traces of insulin using a novel silica nanoparticles-Nafion modified glassy carbon electrode. J. Electroanal. Chem. 2014, 714, 70-75. [CrossRef] 
30. Yunker, P.J.; Still, T.; Lohr, M.A.; Yodh, A.G. Suppression of the coffee-ring effect by shape-dependent capillary interactions. Nature 2011, 476, 308-311. [CrossRef] [PubMed]

31. Gonzalez-Macia, L.; Morrin, A.; Smyth, M.R.; Killard, A.J. Advanced printing and deposition methodologies for the fabrication of biosensors and biodevices. Analyst 2010, 135, 845-867. [CrossRef] [PubMed]

32. Li, J.; Rossignol, F.; Macdonald, J. Inkjet printing for biosensor fabrication: Combining chemistry and technology for advanced manufacturing. Lab Chip 2015, 15, 2538-2558. [CrossRef] [PubMed]

33. Soltman, D.; Subramanian, V. Inkjet-printed line morphologies and temperature control of the coffee ring effect. Langmuir 2008, 24, 2224-2231. [CrossRef] [PubMed]

34. Istamboulie, G.; Sikora, T.; Jubete, E.; Ochoteco, E.; Marty, J.L.; Noguer, T. Screen-printed poly (3,4-ethylenedioxythiophene) (PEDOT): A new electrochemical mediator for acetylcholinesterase-based biosensors. Talanta 2010, 82, 957-961. [CrossRef] [PubMed]

35. Peeters, M.M.; van Grinsven, B.; Foster, C.W.; Cleij, T.J.; Banks, C.E. Introducing thermal wave transport analysis (TWTA): A thermal technique for dopamine detection by screen-printed electrodes functionalized with molecularly imprinted polymer (MIP) particles. Molecules 2016, 21, 552. [CrossRef] [PubMed]

36. Bergamini, M.F.; Santos, D.P.; Zanoni, M.V.B. Determination of isoniazid in human urine using screen-printed carbon electrode modified with poly-L-histidine. Bioelectrochemistry 2010, 77, 133-138. [CrossRef] [PubMed]

37. Moreira, F.T.; Ferreira, M.J.M.; Puga, J.R.; Sales, M.G.F. Screen-printed electrode produced by printed-circuit board technology. Application to cancer biomarker detection by means of plastic antibody as sensing material. Sens. Actuators B Chem. 2016, 223, 927-935. [CrossRef]

38. Reddy, A.S.G.; Narakathu, B.B.; Atashbar, M.Z.; Rebros, M.; Rebrosova, E.; Joyce, M.K. Fully printed flexible humidity sensor. Procedia Eng. 2011, 25, 120-123. [CrossRef]

39. Jia, H.; Wang, J.; Zhang, X.; Wang, Y. Pen-writing polypyrrole arrays on paper for versatile cheap sensors. ACS Macro Lett. 2013, 3, 86-90. [CrossRef]

40. Karim, M.N.; Lee, J.E.; Lee, H.J. Amperometric detection of catechol using tyrosinase modified electrodes enhanced by the layer-by-layer assembly of gold nanocubes and polyelectrolytes. Biosens. Bioelectron. 2014, 61, 147-151. [CrossRef] [PubMed]

41. Petrova, J.; Romanova, J.; Madjarova, G.; Ivanova, A.; Tadjer, A. Absorption spectra of model single chains of conducting polyaniline. J. Phys. Chem. B 2012, 116, 6543-6552. [CrossRef] [PubMed]

42. Bai, H.; Shi, G. Gas sensors based on conducting polymers. Sensors 2007, 7, 267-307. [CrossRef]

43. Ngamna, O.; Morrin, A.; Killard, A.J.; Moulton, S.E.; Smyth, M.R.; Wallace, G.G. Inkjet printable polyaniline nanoformulations. Langmuir 2007, 23, 8569-8574. [CrossRef] [PubMed]

44. Kit-Anan, W.; Olarnwanich, A.; Sriprachuabwong, C.; Karuwan, C.; Tuantranont, A.; Wisitsoraat, A.; Srituravanich, W.; Pimpin, A. Disposable paper-based electrochemical sensor utilizing inkjet-printed Polyaniline modified screen-printed carbon electrode for Ascorbic acid detection. J. Electroanal. Chem. 2012, 685, 72-78. [CrossRef]

45. Rotariu, L.; Istrate, O.M.; Bala, C. Poly (allylamine hydrochloride) modified screen-printed carbon electrode for sensitive and selective detection of NADH. Sens. Actuators B Chem. 2014, 191, 491-497. [CrossRef]

46. Cinti, S.; Neagu, D.; Carbone, M.; Cacciotti, I.; Moscone, D.; Arduini, F. Novel carbon black-cobalt phthalocyanine nanocomposite as sensing platform to detect organophosphorus pollutants at screen-printed electrode. Electrochim. Acta 2016, 188, 574-581. [CrossRef]

47. Dickert, F.L.; Lieberzeit, P.A.; Hayden, O.; Gazda-Miarecka, S.; Halikias, K.; Mann, K.J.; Palfinger, C. Chemical sensors-From molecules, complex mixtures to cells-supramolecular imprinting strategies. Sensors 2003, 3, 381-392. [CrossRef]

48. Poller, A.M.; Spieker, E.; Lieberzeit, P.A.; Preininger, C. Surface imprints: Advantageous application of ready2use materials for bacterial quartz-crystal microbalance sensors. ACS Appl. Mater. Interfaces 2016, 9, 1129-1135. [CrossRef] [PubMed]

49. Schirhagl, R.; Lieberzeit, P.A.; Dickert, F.L. Chemosensors for viruses based on artificial immunoglobulin copies. Adv. Mater. 2010, 22, 2078-2081. [CrossRef] [PubMed]

50. Bajwa, S.Z.; Dumler, R.; Lieberzeit, P.A. Molecularly imprinted polymers for conductance sensing of $\mathrm{Cu}^{2+}$ in aqueous solutions. Sens. Actuators B Chem. 2014, 192, 522-528. [CrossRef]

51. Navakul, K.; Warakulwit, C.; Yenchitsomanus, P.T.; Panya, A.; Lieberzeit, P.A.; Sangma, C. A novel method for dengue virus detection and antibody screening using a graphene-polymer based electrochemical biosensor. Nanomedicine 2017, 13, 549-557. [CrossRef] [PubMed] 
52. Peeters, M.; Troost, F.J.; Mingels, R.H.; Welsch, T.; van Grinsven, B.; Vranken, T.; Ingebrandt, S.; Thoelen, R.; Cleij, T.J.; Wagner, P. Impedimetric detection of histamine in bowel fluids using synthetic receptors with pH-optimized binding characteristics. Anal. Chem. 2013, 85, 1475-1483. [CrossRef] [PubMed]

53. Eersels, K.; van Grinsven, B.R.N.; Khorshid, M.; Somers, V.; Puttmann, C.; Stein, C.; Barth, S.; Diliën, H.; Bos, G.M.J.; Germeraad, W.T.V.; et al. Heat-transfer-method-based cell culture quality assay through cell detection by surface imprinted polymers. Langmuir 2015, 31, 2043-2050. [CrossRef] [PubMed]

54. Quintana, J.C.; Arduini, F.; Amine, A.; Van Velzen, K.; Palleschi, G.; Moscone, D. Part two: Analytical optimisation of a procedure for lead detection in milk by means of bismuth-modified screen-printed electrodes. Anal. Chim. Acta 2012, 736, 92-99. [CrossRef] [PubMed]

55. Wang, J.; Lu, J.; Hocevar, S.B.; Farias, P.A.; Ogorevc, B. Bismuth-coated carbon electrodes for anodic stripping voltammetry. Anal. Chem. 2000, 72, 3218-3222. [CrossRef] [PubMed]

56. Neagu, D.; Arduini, F.; Quintana, J.C.; Di Cori, P.; Forni, C.; Moscone, D. Disposable Electrochemical Sensor to Evaluate the Phytoremediation of the Aquatic Plant Lemna minor L. toward $\mathrm{Pb}^{2+}$ and/or $\mathrm{Cd}^{2+}$. Environ. Sci. Technol. 2014, 48, 7477-7485. [CrossRef] [PubMed]

57. Seekaew, Y.; Lokavee, S.; Phokharatkul, D.; Wisitsoraat, A.; Kerdcharoen, T.; Wongchoosuk, C. Low-cost and flexible printed graphene-PEDOT: PSS gas sensor for ammonia detection. Org. Electron. 2014, 15, 2971-2981. [CrossRef]

58. Wisitsoraat, A.; Pakapongpan, S.; Sriprachuabwong, C.; Phokharatkul, D.; Sritongkham, P.; Lomas, T.; Tuantranont, A. Graphene-PEDOT: PSS on screen printed carbon electrode for enzymatic biosensing. J. Electroanal. Chem. 2013, 704, 208-213. [CrossRef]

59. Truong, L.T.; Chikae, M.; Ukita, Y.; Takamura, Y. Labelless impedance immunosensor based on polypyrrole-Pyrolecarboxylic acid copolymer for hCG detection. Talanta 2011, 85, 2576-2580. [CrossRef] [PubMed]

60. Xin, Y.; Fu-bing, X.; Hong-wei, L.; Feng, W.; Di-zhao, C.; Zhao-yang, W. A novel $\mathrm{H}_{2} \mathrm{O}_{2}$ biosensor based on $\mathrm{Fe}_{3} \mathrm{O}_{4}-\mathrm{Au}$ magnetic nanoparticles coated horseradish peroxidase and graphene sheets-Nafion film modified screen-printed carbon electrode. Electrochim. Acta 2013, 109, 750-755. [CrossRef]

61. Talarico, D.; Arduini, F.; Amine, A.; Cacciotti, I.; Moscone, D.; Palleschi, G. Screen-printed electrode modified with carbon black and chitosan: A novel platform for acetylcholinesterase biosensor development. Anal. Bioanal. Chem. 2016, 408, 7299-7309. [CrossRef] [PubMed]

62. Du, D.; Huang, X.; Cai, J.; Zhang, A. Amperometric detection of triazophos pesticide using acetylcholinesterase biosensor based on multiwall carbon nanotube-chitosan matrix. Sens. Actuators B Chem. 2007, 27, 531-535. [CrossRef]

63. Loaiza, O.A.; Lamas-Ardisana, P.J.; Añorga, L.; Jubete, E.; Ruiz, V.; Borghei, M.; Cabanero, G.; Grande, H.J. Graphitized carbon nanofiber-Pt nanoparticle hybrids as sensitive tool for preparation of screen printing biosensors. Detection of lactate in wines and ciders. Bioelectrochemistry 2015, 101, 58-65. [CrossRef] [PubMed]

64. Weng, B.; Morrin, A.; Shepherd, R.; Crowley, K.; Killard, A.J.; Innis, P.C.; Wallace, G.G. Wholly printed polypyrrole nanoparticle-based biosensors on flexible substrate. J. Mater. Chem. B 2014, 2, 793-799. [CrossRef]

65. Shimomura, T.; Sumiya, T.; Ono, M.; Ito, T.; Hanaoka, T.A. A novel, disposable, screen-printed amperometric biosensor for ketone 3 - $\beta$-hydroxybutyrate fabricated using a 3- $\beta$-hydroxybutyrate dehydrogenase-mesoporous silica conjugate. Anal. Bioanal. Chem. 2013, 405, 297-305. [CrossRef] [PubMed]

66. Lesch, A.; Cortés-Salazar, F.; Amstutz, V.; Tacchini, P.; Girault, H.H. Inkjet printed nanohydrogel coated carbon nanotubes electrodes for matrix independent sensing. Anal. Chem. 2015, 87, 1026-1033. [CrossRef] [PubMed]

(C) 2017 by the author. Licensee MDPI, Basel, Switzerland. This article is an open access article distributed under the terms and conditions of the Creative Commons Attribution (CC BY) license (http://creativecommons.org/licenses/by/4.0/). 